EDITORIAL

\title{
MÉMOIRE ET SOUVENIR EN AFRIQUE ET DANS LA DIASPORA
}

\author{
Daniela Merolla \\ Institut National des Langues et Civilisations Orientales, \\ Université Sorbonne Paris-Cité, France
}

La littérature peut être considérée comme un «lieu de mémoire» particulier. Les conteurs et les écrivains sont des utilisateurs et des producteurs de souvenirs, et la littérature orale et écrite ainsi que le cinéma sont des moyens puissants pour créer une mémoire collective « en se rappelant le passé sous forme de récit 》-comme le soulignent Astrid Erll et Agnes Rigney (2006 : 112). Ce numéro spécial de la revue Afrika Focus comprend des articles qui illustrent de manière frappante le rôle de la littérature orale et écrite et des films dans la transmission et l'actualisation de la mémoire culturelle en Afrique. Ces articles proviennent d'une sélection des communications présentées à la 11ème conférence d'ISOLA (Société internationale pour les littératures orales d'Afrique) qui s'est tenue à l'université de Floride, à Gainesville, aux États-Unis, du 25 au 28 mai 2016.

Comme indiqué par Tunde Akinyemi (2016) dans l'argumentaire du colloque, la mémoire culturelle et celle individuelle s'entrecroisent et se nourrissent réciproquement : «bien que l'individu soit le medium exclusif par lequel la mémoire se présente, la mémoire que l'individu "porte" est toujours définie par des facteurs sociaux». Plusieurs études sur la mémoire culturelle montrent effectivement que se rappeler du passé n'est pas simplement un acte individuel, mais un processus façonné par les réseaux sociaux et les modèles culturels, ce qui influence la manière dans laquelle des éléments du passé sont représentés pour des objectifs présents et futurs ${ }^{1}$.

Les formes que la mémoire culturelle peut prendre sont nombreuses. L'historien Pierre Nora (1989) a utilisé en premier la définition de «lieux de mémoire» mentionnée cidessous pour se référer à des processus matériaux et immatériaux mis en ouvre par les institutions étatiques pour créer les identités nationales à travers la sélection, l'oubli, voire l'invention d'événements significatifs ${ }^{2}$. La mémoire nationale prend forme à travers «la matérialité de la trace» (Nora, 1989), telle que des monuments commémoratifs, des

1 Halbwachs, 1950 ; Nora, 1989 ; Erll, Nünning et Young, 2008 ; Dermentzopoulos et Kosmidou, 2016.

2 Assmann $1995: 129$. 
sanctuaires, des musées, des archives et des lieux emblématiques, et à travers des formes moins matérielles comme celles de discours, d'anniversaires, de cérémonies et de rituels. Les souvenirs collectifs sont structurés par tous ces lieux de mémoire et deviennent l'histoire officielle et le discours dominant de la nation. La mémoire culturelle peut également se confondre avec la notion de "patrimoine», ce qui indique une autre caractéristique centrale de la mémoire : son investissement émotionnel. Selon l'historienne Luisa Passerini (2008), nous devrions effectivement étudier la valeur des émotions dans la création et la transmission de souvenirs entre générations et entre groupes culturels, ce qu'elle appelle «l'intersubjectivité de la mémoire». Finalement, si se rappeler du passé est au cœur de la construction de la mémoire collective - et l'affectivité y joue un rôle structurant -, l'oubli apparaît également important. Connerton (2008: 60 et 63) note que l'oubli joue un rôle non seulement dans les formes répressives de la damnatio memoriae, l'effacement de faits et de personnes de la mémoire publique, mais également dans la formation de nouvelles identités par des mémoires inédites ainsi que des silences tacitement partagés. Nous construisons, transmettons et même manipulons ce qu'il faut retenir et comment le retenir à la lumière des configurations présentes et futures de notre identité. Les différences et les oppositions dans ce que nous rappelons, comment nous nous souvenons, et ce que nous faisons de ces souvenirs en tant que groupe conduisent à des situations dans lesquelles la mémoire collective et culturelle est le plus souvent un champ de bataille. Les souvenirs sont controversés, car la mémoire du passé parle des rapports de force et des déséquilibres du présent comme de l'espoir et des craintes pour le futur.

La forme du récit - oral, écrit ou (audio)visuel - est un outil formidable pour développer le dialogue entre souvenirs individuels et mémoires collectives. Et si les «sites de mémoire» forment souvent un discours hégemonique et conventionnel, le récit peut aussi donner voix aux souvenirs réduits au silence dans ces histoires dominantes. Nous pensons par exemple aux «souvenirs vécus», ceux des expériences personnelles et des événements dont la mémoire est transmise du détenteur du souvenir aux générations suivantes. Plus complexe est le cas des genres oraux qui racontent le passé et l'adaptent au présent - comme le font souvent les mythes et les récits épiques. Ils peuvent être des «sites de mémoire» lorsqu'ils construisent et confirment les structures sociales existantes et une histoire dominante (par exemple celle des conquérants locaux ou des perspectives masculines par rapport aux perspectives féminines), mais ils peuvent également assumer une fonction alternative. Par exemple, quand ils sont porteurs d'un discours local par rapport à la mémoire créée par le colonialisme et par le nationalisme de la période postcoloniale. Dans ce cas, les récits oraux peuvent faire place à des épisodes anéantis par les histoires nationales et par les représentations hégémoniques du passé, et ainsi exprimer des histoires et des perspectives oubliées, comme dans le cas des représentations locales des rencontres coloniales ainsi que postcoloniales dans le processus de plus en plus avancé de la mondialisation.

Les articles réunis dans ce volume présentent des formes variées de mémorisation par la littérature et l'audiovisuel. La mémoire collective de l'oralité et de son rôle dans la pratique esthétique du langage et dans l'identité de la communauté, en se reliant au souvenir 
affectif des genres oraux, est traitée par exemple dans les textes d'Abidemi Bolarinwa (Recreation of Oral Poetic Genres in Selected Yorùbá Home-Video Films/ La recréation de genres poétiques oraux dans une selection de films vidéo Yorùbá), d'Adekemi Agnes Taiwo (New Media, Old Artistry : The Adaptation of Yorùbá Folktale. Narrative Strategies in Video Films/ Nouveau média, talent ancien : adaptation du conte folklorique yorùbá. Stratégies narratives des films vidéo), ainsi que par Chigozie Bright Nnabuihe et Adaora Lois Anyachebelu (Reorientation for National Transformation : the Role of Literature/ Réorientation pour la transformation nationale : le rôle de la littérature).

Dans l'article de Paulette Roulon-Doko (Une mémoire méconnue : témoignages sur des attaques de 2006 en zones rurales en République Centrafricaine / An unrecognized memory : testimonies on attacks of 2006 in rural areas in the Central African Republic), il s'agit de la formation de la mémoire culturelle par des témoignages qui présentent des «souvenirs vécus», et qui permettent ainsi la transmission d'un savoir autour de l'expérience des pillages pour mieux se protéger à l'avenir.

L'utilisation des genres oraux par plusieurs médias et la complexité de leur mémorialisation progressive par des agents différents sont analysées par Tal Tamari dans le cas d'un récit épique (Salimu : From Memory to Literary Narrative in Twentieth-Century Manding Culture / Salimu : De la mémoire au récit littéraire dans la culture mandingue du XXe siècle) et par Abubakar Aliyu Liman dans le cas d'une légende (Memorializing a legendary figure : Bayajidda the prince of Bagdad in Hausa Land / Commémoration d'une figure légendaire : Bayajidda, prince de Bagdad, en pays haoussa).

Ensuite, la formation des nouvelles mémoires culturelles par le rapport intertextuel entre oralité et littérature écrite contemporain se retrouve dans les articles d'Edoama Odueme (Orality, Memory and the New African Diaspora Poetry : Examining Tanure Ojaide's Poetics / L'oralité, la mémoire et la nouvelle poésie de la diaspora africaine : examen de la poétique de Tanure Ojaide), de Rasheedah Liman (History and Dramatic Imagination in Ahmed Yerima's Attahiru / Histoire et imaginaire dramatique dans Attahiru d'Ahmed Yerima), et de Bosede Funke Afolayan (The Court Poet/Praise Singer in Wole Soyinka's Death and the King's Horseman and Ola Rotimi's Ovonramwen Nogbaisi : A Critical Appraisal / Le poète de cour/chanteur de louanges dans La Mort et le cavalier du roi de Wole Soyinka et Ovonramwen Nogbaisi d'Ola Rotimi : une évaluation critique) qui soulève aussi la mise en question de la mémoire culturelle dominante grâce à l'utilisation des sources orales.

L'article final de Rémi Armand Tchokothe (Archiving collective memories and (dis) owning / Archivage des mémoires collectives et (dé)possession) porte sur la réflexion autour de la propriété de la mémoire collective à l'ère digitale. En se référant à l'idée de «chercheurs comme de griots», Tchokothe invite les scientifiques à repenser leur position hégémonique par rapport à ceux qui leur donnent leur savoir, leurs témoignages, et leurs interprétations pendant le travail de terrain, en plaidant pour le développement de mesures protectrices et proactives pour contrer le risque de la damnatio memoriae des voix locales par l'archivage électronique. 


\section{Bibliographie}

Akinyemi, Tunde. 2016. «Argumentaire». 11ème conférence d'ISOLA (Société internationale pour les littératures orales d'Afrique). Université de Floride, Gainesville, États-Unis, 25 - 28 mai. https:// africaisola.org/past-conferences-conferences-passees

Assmann Jan (1995). «Collective memory and cultural identity». New German Critique, vol. 65, pp. 125133. Traduction de John Czaplicka.

Dermentzopoulos Christos et Rania Kosmidou (2016). «Introduction : Special issue : Studies in cultural memory ». International Journal of Media \& Cultural Politics, vol. 12 (1), pp. 3-6.

Erll Astrid et Ansgar Nünning en coll. avec Sara B. Young (dir. ) (2008). Cultural Memory Studies : An International and Interdisciplinary Handbook. Berlin/New York, De Gruyter.

Erll Astrid et Agnes Rigney (2006). «Literature and the production of cultural memory : Introduction». European Journal of English Studies, vol. 10 (2), pp. 111-115.

Halbwachs Maurice (1950). La mémoire collective. Paris, Presses Universitaires de France.

Nora Pierre (1989). «Between memory and history : Les Lieux de Mémoire». Representations - Special Issue : Memory and counter-memory, vol. 26, pp. 7-24. 